\title{
DOCUMENTS
}

Werner Blumenberg

\section{MARX' UND ENGELS' BRIEFWECHSEL MIT FRANZ DUNCKER}

Mit Franz Duncker (1 822-I 888), dem liberalen Politiker, Verleger und Besitzer der Berliner Volkszeitung, stand Lassalle seit der Veröffentlichung seines Werkes über die Philosophie Heraklits durch Dunckers Verlag im Jahre 1858 bis Anfang des Jahres $\mathrm{r} 86 \mathrm{I}$ in freundschaftlichen Beziehungen. ${ }^{1}$ Lassalle stellte auch für Marx und Engels die Verbindung zu diesem Verlage her. Hier erschienen Marx' „Zur Kritik der Politischen Ökonomie" und Engels” Broschüre „Po und Rhein”, beide im Jahre 1859 .

Als Lassalle hörte, dass die Krise des Jahres I 857 Marx dazu angespornt habe, sich nun ernsthaft an die Ausarbeitung seiner Grundzüge der Ökonomie zu machen, bot er seine Hilfe an, falls das Werk in einem Berliner Verlag erscheinen solle. ${ }^{2}$ Marx unterrichtete Lassalle über den Stand der Arbeit. Mit der endgültigen Ausarbeitung sei er seit einigen Monaten beschäftigt; aber sie schreite sehr langsam vorwärts, da Gegenstände, die man seit vielen Jahren studiert habe, immer neue Seiten zeigten und neue Bedenken erweckten, sobald man die Arbeit abschliessen wolle. Am zweckmässigsten erschien ihm die Herausgabe in zwanglosen Heften. Zunächst handle es sich um die Kritik der ökonomischen Kategorien, und am liebsten würde er das Material stark kondensieren; denn in Heften würde alles breiter. ${ }^{3}$ Das ganze Werk werde kaum weniger als dreissig bis vierzig Bogen umfassen müssen. Die erste Lieferung, die „I. Wert, 2. Geld, 3. Das Kapital im allgemeinen (Produktionsprozess des Kapitals, Zirkulationsprozess des Kapitals, Einheit von beiden oder Kapital und Profit, Zins)" umfasse, bilde eine ,,selbständige Broschüre”, die nicht kürzer als fünf bis sechs Bogen sein könne. Der Verleger würde dies

\footnotetext{
1 Über diese hier nicht interessierenden Beziehungen und ihren durch politische Meinungsverschiedenheiten herbeigeführten Abbruch s. Gustav Mayers Einleitung zum zweiten Bande der „Nachgelassenen Briefe und Schriften” Lassalles, Berlin 1923, S. 22 ff. Die im folgenden erwähnten Briefe zwischen Marx-Engels und Lassalle sind nach dem dritten Bande dieser Ausgabe, Berlin i922, angeführt.

2 Marx an Lassalle 21. Dezember 1857; Lassalle an Marx ro. Februar i858.

${ }^{3}$ Marx an Lassalle 22. Februar 1858.
} 
Manuskript Ende Mai haben können. ${ }^{1}$ Es gelang Lassalle, einen vorteilhaften Vertrag mit Duncker darüber abzuschliessen. Aber Ende Mai musste Marx mitteilen, dass ihn Krankheit an der Ausarbeitung gehindert habe. Ende Juli schreibt Lassalle, dass sie täglich auf das Manuskript gewartet hätten, und Ende Oktober findet er es unerklärlich, dass noch immer nichts bei Duncker eingetroffen sei. Marx entschuldigt die Verzögerung mit Krankheit und journalistischer Erwerbsarbeit. „Der eigentliche Grund ist aber der: Der Stoff lag vor mir; es handelte sich nur noch um die Form..." In vier Wochen werde er fertig sein; mit dem Schreiben habe er jetzt eigentlich erst angefangen. Ausserdem werde „die erste Abteilung ,Das Kapital im allgemeinen' gleich zwei Hefte einnehmen”.2 Am 28. März 1859: „Du wirst sehen, dass die erste Abteilung das Hauptkapitel, nämlich das dritte vom Kapital, noch nicht enthält. Ich hielt dies geraten aus politischen Gründen; denn mit C[apitel] 3 beginnt die eigentliche Schlacht, und es schien mir ratsam, nicht erschrecken zu machen de prime abord."3 Ende Januar traf das Manuskript in Berlin ein, und gegen Mitte Juni erschien das Werk.

Wir wissen heute, da der Marx I 858 vorliegende „Stoff” zugänglich ist $^{4}$, dass es keineswegs nur Fragen der Form waren oder die Reservierung des Kapitels vom Kapital für eine spätere Lieferung ,aus politischen Gründen", welche die Fertigstellung des Manuskriptes verzögerten, sondern dass die Ursache der Verzögerung das Ringen mit dem Stoff selbst war. Diese erste Lieferung beschäftigte sich nur mit den Kategorien der Ware und des Geldes; ihr liegen zugrunde „Das Kapitel vom Geld” und ein Fragment der Urfassung der „Kritik" vom Jahre 1858, S. 35-148 bzw. 871-974 der „Grundrisse”. Es wat eine erstaunliche Arbeitsleistung, in wenigen Wochen aus diesem Material das dann veröffentlichte Werk zu gestalten.

In Marx' Briefwechsel mit Duncker werden nur verlegerische Fragen besprochen. Er wurde von Marx in einer überreizten Stimmung geführt, die wahrscheinlich durch Krankheit und Überarbeitung verschärft wurde und die Marx' normales Misstrauen noch erheblich

1 Marx an Lassalle ix. März I8 58 .

Marx an Lassalle 12. November I 858 .

- Uber die Entstehung des Werkes und die Änderung des Arbeitsplanes s. a. D. B. Rjazanov: Sicbzig Jahre ,Zur Kritik der politischen Okonomie”, in: Grünberg, Archiv, XV. Jahrg. 1930, Heft I S. lff. - Rjazanov waren Marx' Briefe an Duncker noch unbekannt.

" "Grundrisse der Kritik der Politischen Ökonomie (Rohentwurf). I 857-18 8 8", zwei Bde, Moskau 1939-4I; neue Ausgabe Berlin, Dietz Verlag, 1953. Dazu A. I. Malyschs Würdigung und Analyse „Marx' ökonomische Manuskripte von $1857 / 58$ - eine wichtige Etappe in der Entwicklung der marxistischen politischen Ökonomie", in: Beiträge zur Geschichte der deutschen Arbeiterbewegung, 5. Jahrg. 1963, Heft ${ }_{3}$ S. 42 Iff, 
steigerte. Der Druck des Manuskriptes wurde hinausgeschoben, da Duncker mit dem Druck eines Lassalleschen Dramas beschäftigt war, das Marx am 6. März erhielt. Später wurde die Herstellung wiederholt unterbrochen, da Duncker aktuelle, an den Tag gebundene Broschüren herausgab: Engels' Broschüre „Po und Rhein” erschien am 5. April, und Lassalles Broschüre „Der italienische Krieg und die Aufgabe Preussens" kam Ende Mai heraus und erlebte bereits nach zwei Wochen eine zweite Auflage. Das alles war nicht geeignet, Marx' Empörung über die Verzögerung des Druckes zu beschwichtigen. Dazu kam, dass Marx aus Amerika die Mitteilung erhielt, eine grössere Anzahl von Exemplaren sei bestellt und eine weit grössere Bestellung zu erwarten, sobald Umfang und Preis des Werkes bekannt seien. ${ }^{1}$ Das macht Marx' Andringen darauf bei Duncker verständlich.

Der Briefwechsel endete mit einem Missklang; Duncker bedauerte fast, dass Lassalle überhaupt die Verbindung mit Marx angeknüpft habe. Trotzdem gelang es Lassalle, Duncker auch zur Übernahme des zweiten Heftes in seinen Verlag zu bewegen, falls es nicht stärker als vier bis fünf Bogen sei. Aber wenn es auch viel stärker werde, setzte Lassalle hinzu, solle Marx das Manuskript schicken; er werde dann das Weitere besorgen ${ }^{2}$. Marx meinte Anfang Juli, dieses Heft werde kaum vor Ende Dezember fertig werden. Lassalle mahnte Ende Januar I 860, warum die Fortsetzung nicht komme, die für spätestens Ende Dezember versprochen sei. „Also schicke das Manuskript an Duncker oder schreib mir mindestens, was Dich hindert." Marx antwortete am 30. Januar: „Meine nationalökonomische Schrift, - wenn Heft II erschienen, enthält erst Schluss von Abteilung I, Buch I, und es sind sechs Bücher..." Dass das Heft noch nicht in Berlin eingetroffen sei, sei ,die Schuld scheusslicher Umstände”.

Zu dieser Zeit nahm die Vogt-Affäre Marx bereits sehr in Anspruch, und es folgten unangenehme Auseinandersetzungen mit Lassalle, nachdem Marx ihm anonyme Verdächtigungen zugesandt hatte. Aber es unterliegt kaum einem Zweifel, dass auch ohne diese Ereignisse das Erscheinen des Werkes in weiteren Heften nicht fortgesetzt wer-

1 S. S. I I I Anm. 2. Es lässt sich nicht feststellen, ob es sich hierbei um eine wirkliche Bestellung handelte; Duncker erwähnt sie im Brief Nr. 9 nicht, sondern gibt nur an, dass neben Buchhandlungen in Deutschland auch zwei Buchhandlungen in New York Exemplare erhielten. A. Komp, der Absender des Briefes, war stellvertretender Vorsitzender des 1857 bis 1867 in New York bestehenden Kommunistenklubs; er kehrte einige Monate nach der Absendung des Briefes nach Deutschland zurück. Fr. Kamm, der Vorsitzende des Klubs, hatte Marx am Io. Dezember I 857 mitgeteilt, der Klub zähle dreissig Mitglie der. Wie gross die Mitgliederzahl Anfang I 859 war, ist nicht bekannt.

2 Lassalle an Marx I1. Juli 1859. 
den konnte. Wieder lag die Unmöglichkeit, das Manuskript zu liefern, im „Stoff”. Das „Kapitel vom Kapital”, das Thema des geplanten zweiten Heftes, umfasst in den „Grundrissen" mehr als fünfhundert Seiten und eine grosse Anzahl Exkurse. Unmöglich war es, daraus ein Heft von einigen Bogen zu machen. Marx gab bald den Gedanken an die Veröffentlichung in dieser Form auf. Dieses Kapitel wurde später das ganze „Kapital”, das Marx selbst im Vorwort des von ihm im Jahre I 867 veröffentlichten ersten Bandes als die Fortsetzung der i 859 veröffentlichten Schrift „Zur Kritik der Politischen Ökonomie” bezeichnete.

Dies ist die tiefere Ursache der Nichtfortsetzung der Publikation in Heften. Auch die Aufnahme des Werkes konnte Marx kaum zur Fortsetzung ermutigen. Ungeduldig erwartete er Ankündigungen und Besprechungen ${ }^{1}$. Grundlos verdächtigte er Lassalle, er wolle ihn totschweigen, nachdem er früher die Fertigstellung des Druckes verzögert habe. ${ }^{2}$ Als drei Wochen nach dem Erscheinen noch kein Echo in der Öffentlichkeit zu vernehmen war, drohte er, er werde die Fortsetzung nur noch englisch schreiben, - eine Ankündigung, die Lassalle mit Humor aufnahm. ${ }^{3}$ Selbst im engeren Kreise konnte das Werk nicht sensationell wirken. Liebknecht äusserte sich darüber, niemals habe ihn ein Buch mehr enttäuscht als dieses. ${ }^{4}$ Die Parteifreunde hatten gewiss ein Werk erwartet, das sozialistische Heilmittel bot und propagandistisch zu verwerten war; statt dessen erhielten sie ein schwerverständliches Buch, das einige ökonomische Kategorien wissenschaftlich behandelte.

Auch der Briefwechsel zwischen Engels und Duncker behandelte verlegerische Fragen. Engels' Briefe vom 25. Juli 1859 und 6. Februar I 860 sind nicht erhalten ${ }^{5}$; aber ihr Inhalt ergibt sich aus Dunckers präzisen Antworten und aus Engels' Korrespondenz mit Lassalle. Dieser hatte auch für die Herausgabe der Broschüre „Po und Rhein” den Dunckerschen Verlag bereit gefunden. Dankbar begrüsste es Engels, dass ihm dadurch nach zehn Jahren wieder die Gelegenheit geboten werde, vor dem deutschen Publikum zu erscheinen. ${ }^{6}$

Engels bestritt in seiner Broschüre über den österreichisch-französischen Konflikt die Auffassung weiter Militärkreise, dass Deutschland des Po und Mincio als natürlicher Grenzen zu seiner Verteidigung bedürfe; denn mit demselben Rechte könne Frankreich das linke

1 Marx an Engels 18. Juli, 22. Juli 1859.

2 Marx an Engels ro. März, 24. Mai, 25. Mai, 7. Juni, ro. Dezember 1859; Marx an Kugelmann 28. Dezember I 862.

3 Marx an Lassalle Anfang Juli, Lassalle an Marx I r. Juli 1859.

4 Marx an Engels 22. Juli i 859.

5 Engels' Brief vom 20. Februar in Marx-Engels, Werke, Bd. 30, Berlin 1964, S. 454.

- Engels an Lassalle I4. März I859. 
Rheinufer als natürliche Grenze zu seiner Verteidigung fordern. Er wünschte gleichwohl die Unterstützung Österreichs, da Napoleons Cäsarismus der Gegner der revolutionären Bewegung sei, hinter dem noch als weit gefährlicherer Gegner der russische Zarismus stehe. Lassalle vertrat demgegenüber in seiner Broschüre „Der italienische Krieg und die Aufgabe Preussens" die Auffassung, dass die Zwangslage Österreichs auszunutzen sei zur Einigung Deutschlands unter preussischer Führung; es war die politische Linie des fortschrittlichsten Teiles des Nationalvereins und der norddeutschen Demokraten. Im Briefwechsel Marx - Engels - Lassalle nimmt die Diskussion dieser gegensätzlichen Ansichten einen breiten Raum ein.

Als Engels nach der Annexion Savoyens und Nizzas durch Frankreich eine neue Broschüre „Savoyen, Nizza und der Rhein" verfasste, machte Duncker ihre Veröffentlichung davon abhängig, dass ihr keine „prinzipiellen Bedenken” entgegenständen. ${ }^{1}$ Duncker war inzwischen selbst in der Eisenacher August-Versammlung des Nationalvereins hervorgetreten, und seine Zeitung vertrat dessen Linie nachdrücklich, insbesondere nach dem Erscheinen der Lassalleschen Broschüre, so dass Duncker eine Stärkung des pro-österreichischen Kurses durch seinen Verlag kaum zulassen konnte. Nachdem Duncker die Veröffentlichung bereits abgelehnt hatte, erreichte es Lassalle, dass er sich doch noch zum Druck bereit erklärte, falls Engels sie nicht anonym, sondern unter seinem Namen erscheinen lassen wolle. Lassalle musste anerkennen, dass ein Verleger beim Verlage anonymer politischer Broschüren eine Solidarität mit deren Tendenz zugebe. ${ }^{2}$ Die Broschüre erschien dann anonym im Verlag G. Behrend, Berlin.

\section{DUNCKER AN MARX.}

\section{Geehrter Herr!}

Berlin, 7. Februar 1859 .

Hierdurch bestätige ich den richtigen Empfang Ihrer M[anu]sk[ri]ptsendung vom 26. v. Mts, und zwar ist damit alles in bester Ordnung gegangen. Wie der Poststempel nachweist, und wie Ihre Benachrichtigung von hier gleichfalls lautete, ist das Paket am 3 I. hier angekommen, am Montag, heute vor acht Tagen, und auch noch am selben Tage oder am Dienstag früh, das kann ich jetzt nicht mehr genau feststellen, hatte ich den Postschein darüber in Händen; denn als Wertstück und

1 Engels sah im Brief an Lassalle vom 20. Februar 1860 diese „prinzipiellen Bedenken” darin, dass die in seiner Broschüre vertretene Auffassung der Lassalles widerspreche, und in verdeckter Form verdächtigte er, zu Unrecht, Lassalle, dass er die Unterdrückung der Broschüre daher vielleicht selbst wünschen könne.

2 Lassalle an Marx und Engels Ende Februar 1860. 
vom Ausland kommend, musste dasselbe nach den reglementarischen Bestimmungen von der Post durch meine Leute abgeholt werden, so erhielt ich dasselbe am Dienstag, den I. Februar vormittags, und es war ein Zufall, dass Ihnen gerade Lassalle am 31. schreiben musste, das M[anu]sk[ri]pt sei noch nicht eingetroffen. ${ }^{1}$ Diese Woche bin ich noch mit einer Drucksache für Lassalle sehr beschäftigt ${ }^{2}$, in der nächsten wird dann der Druck Ihres M[anu]sk[ri]ptes in Angriff genommen werden.

\section{Hochachtungsvoll und ergebenst \\ Franz Duncker.}

2. MARX AN DUNCKER.

London, 23. Febr[uar] I 859 .

Werter Herr, Einliegend erfolgt das Vorwort. ${ }^{3}$

Ihr ganz ergebener

Karl Marx.

3. MARX AN DUNCKER.

[London], i7 March 1859 .

Werter Herr,

Ich schicke die Korrekturbogen im Brief kuvert zurück, weil mir von der hiesigen Post angezeigt worden, dass Korrekt[uren] von Berlin nach London geschickt werden können, den Weg von London nach Berlin aber als Briefe machen müssen.

Seite 32 in den letzten Zeilen habe ich vergessen, statt ,tieferer ökono-

${ }^{1}$ Lassalle an Marx 31. Januar: „Ich begreife nicht, warum Dein Manuskript noch immer nicht ankommt... Ich schreibe Dir daher nochmals, um Dir zu sagen, dass wenn es nun nicht baldigst kommt, Du Dir selbst im Wege stehst, insofern es dann wieder bis zum Herbst warten muss...” Marx an Lassalle 2. Februar: „Das Manuskript ging ab von hier am 26. Januar, und am 31. Januar war bier schon die Anzeige zurück von Berlin, dass das Manuskript angekommen... Unter allen Umständen hat also die preussische Regierung - vielleicht Freund Stieber - drei Tage das Manuskript durchgestöbert... In ihrem eigenen Interesse setze ich voraus, dass die preussische Regierung keine false steps mit meinem Manuskript gemacht. Sonst würde ich in der Londoner Presse (Times etc.) ein Ungewitter vom Teufel veranstalten.” Marx an Lassalle 4. Februar: „Von Herrn Duncker ist noch keine Empfangsanzeige eingetroffen, und es bleibt mir so noch unsicher, ob das Manuskript schon die Hände der Bebörden verlassen hat..."

"Sein Drama „Franz von Sickingen."

${ }^{3}$ Es ist datiert ,im Januar I 859 " und enthält Marx' Darstellung seines Studienganges und die berühmte Fassung der Grundgedanken seiner Geschichtsauffassung. 
mischer Gegensatz zwischen engl[ischer] und fr[an]z[ösischer] etc. Ökonomie" zu korrigieren ,tieferer prinzipieller etc”. ${ }^{1}$

Es ist möglich, dass mich mein Gedäcbtnis täuscht, aber es scheint mir, als ob die Anfänge der Sätze von S. 33, sowohl im Text als der ersten Note, auf Seite 32 gefehlt hätten.

Ihr ganz ergebener

K. M.

\section{MARX AN DUNCKER.}

London, 21. Mai I859.

Werter Herr,

Aus einem von Nordamerika mir zugekommenen Schreiben ersehe ich, dass ungefähr roo Exemplare auf das erste Heft unter meinen Parteifreunden bestellt sind. Ob Sie die Bestellung bereits erhalten, weiss ich nicht. Gleichzeitig aber wird mir angezeigt, dass auf roo Exemplare mehr in denselben Kreisen zu rechnen, sobald der Preis bekannt. ${ }^{2}$ Ich ersuche Sie also, umgebend mir letzteren anzuzeigen. Es schliesst dies natürlich nicht spätere Anzeige der Schrift in London für das allgemeine Publikum aus. Die Langsamkeit, womit die Sache betrieben wird, scheint mir nicht in Ihrem eignen Interesse. In meinem ist sie sicher nicht. In das Druckfeblerverzeichnis der mir zugekommenen Bogen ersuche ich Sie noch aufzunehmen:3

\footnotetext{
${ }^{1}$ Der Text lautet: „....springt hier jedoch zugleich der tiefere principielle Gegensatz hervor, der sich als beständiger Kontrast zwischen ächt englischer und ächt französischer Oekonomie wiederholt."

2 Albrecht Komp an Marx, New York 24. April: „....Es freut mich daher, dass ich bereits mehr als 85 Subskribenten auf Thre Kritik der politischen Ökonomie gesammelt habe - und ich würde deren über 200 zählen können, wenn ich endlich das Nähere über Preis und Umfang des Werkes angeben könnte. Wenn uns nur der verdammte Buchhändler nicht im Stich lässt...” In einer Nachschrift: „Sobald meine Abreise beschlossen und deren Zeit feststeht, werde ich Sie davon benachrichtigen. Bitte schreiben Sie bald und sagen Sie, ob Sie Aussicht haben, Ihr Werk bald gedruckt zu sehen..." - Joseph Weydemeyer an Marx, Milwaukee 27. März: „...Ich habe sofort nach Empfang Deiner Zeilen vom I. Februar alles in Bewegung gesetzt, um Herrn Duncker die Überzeugung beizubringen, dass Dein Werk über die politische Ökonomie auf eine weite Verbreitung zu rechnen hat. Die Zahl der Abonnenten von unserem Kontinente wird freilich niemals so gross werden, um quantitativ einen Ausschlag geben zu können... New York ist natürlich der Hauptplatz, und Komp ist der beste und tïchtigste Agent, den Du dort finden konntest; wie er mir gestern schrieb, hat er bereits 85 Subskribenten auf seiner Liste..." In Chicago sei auf etwa 10, in Milwaukee auf kaum mehr als 6 Subskribenten zu rechnen. (Im Marx-Nachlass). Da Weydemeyers Brief dem Komps beigelegt wurde, s. Marx an Engels 18. Mai, dürfte er 27. April statt März zu datieren sein.

${ }^{3}$ Der Schluss des Briefes ist abgeschnitten.
} 
5. MARX AN DUNCKER. ${ }^{1}$

[Von Dunckers Hand:] Freitag eingetroffen.

Werter Herr,

London, 28. Mai I859.

Auf meinen Brief, worin ich Sie ersuchte, mir den Preis des Heftes zu schreiben, fanden Sie passend nicht zu antworten. Da die Post nach Amerika nur zweimal in der Woche geht, wurde ich so verhindert, meinen Freunden zu antworten.

Als Sie mein M[anu]sk[ri]pt erhielten, dauerte es 14 Tage, bis ich Empfangsanzeige bekam. Darin hiess es, nach einer Woche solle der Druck begonnen werden. Aus der Woche wurden mehr als 3 Wochen. Vor ungefähr 8 Wochen schrieb Lassalle, Mitte Mai werde die Sache fertig sein. ${ }^{2}$ Vor mehr als 3 Wochen erhielt ich die 3 letzten Korrekturbogen. Die Veränderungen, die zu machen waren, konnten bequem in einem Tage bewerkstelligt werden. Statt dessen scheint während dieser ganzen Zeit, obgleich der Druck fertig, wieder völlige Unterbrechung eingetreten. Ich erkläre Ihnen hiermit, dass ich dieser systematischen und planmässigen Verschleppung müde bin $^{3}$ und dass ich Sie hiermit auffordere, und zwar kategorisch, ein Ende mit diesen Manövern zu machen, deren Zweck mit höchst verdächtig scheint. Alle meine Bekannten in England teilen meine Ansicht und haben mich selbst dringend aufgefordert, diesen letzten Schritt zu tun.

Ihr ergebener

$$
\text { Dr. K. Marx. }
$$

\section{DUNCKER AN MARX. ${ }^{4}$}

Geehrter Herr!

Berlin, 3 1 . Mai I 859 .

In umgehender Erwiderung Ihrer Zeilen vom 28. Mai, welche mir heute zugegangen sind, bemerke ich rücksichtlich Ihrer für mich verletzenden Unterstellung einer planmässigen Verschleppung, Manövern u. dgl., dass von einer solchen auch nicht im entferntesten die Rede sein kann. Wenn Sie die Verhältnisse einer deutschen Verlagshandlung noch nicht ganz aus dem Gedächtnis verloren haben: so

${ }^{1}$ Dies ist Marx' ,saugrober Brief nach Berlin”, den er Engels am 24. Mai ankündigte.

2 Lassalle an Marx 8. April: „... Übrigens sagte mir Duncker hierbei, dass Dein Honorar Mitte Mai fällig sein würde."

${ }^{3}$ Im P.S. des langen Briefes an Marx und Engels vom 27. Mai, das also nach dem Eintreffen dieses Briefes in Berlin geschrieben wurde, sagt Lassalle: „In drei Tagen erscheint Dein Werk, lieber Marx. Du hast mich in rechte Verlegenheit gebracht, dem Buchhändler von 'planmässiger' Verschleppung zu schreiben...” Er ,,war ebenso verwundert wie verletzt, als er derartige Vorwürfe gegen sich erhoben sah."

${ }^{4}$ Konzept auf der Rückseite des vorigen Briefes. 
werden Sie begreifen, dass eine solche ein derartiges Werk wie das Ihre neben andern fortlaufenden Unternehmungen und Sachen, die oft von der augenblicklichen Zeitströmung abhängen, herzustellen pflegt, und dass daher allerdings manchmal Verzögerungen eintreten können und müssen. Die letzte wurde verursacht neben andern Verhältnissen durch das Erscheinen zweier Ihnen wahrscheinlich bekannt gewordenen Broschüren: „Po und Rhein” und „Der italienische Krieg und die Aufgabe Preussens". ${ }^{1}$ Um diese rasch auf den Markt zu werfen, habe ich alle andern Sachen beiseitegesetzt, bei denen weder eine bestimmte Verpflichtung, zu einer bestimmten Zeit fertig zu sein, noch eine Gefahr im Verzug vorlag; beides war bei Ihrem Werk nicht vorhanden.

Ausserdem ist in diesem Monat die Leipziger Buchhändlermesse mit ihren diesmal sehr traurigen Verhältnissen gehalten, die meine Zeit völlig in Anspruch genommen hat; dies auch der Grund, warum Ihr letzter Brief keine Antwort gefunden, weil ich abwesend war. Ich kann diese Verzögerung in Ihrem Interesse bedauern, ein Vorwurf trifft mich nicht im mindesten, und ich muss daher diesen wie die sämtl[ichen] direkten und indirekten Ihres letzten Schreibens auf das entschiedenste zurückweisen.

Ihr Buch soll nunmehr in der nächsten Woche ausgegeben werden, und werde ich Ihnen dann sofort den Rest des Honorars, da Sie rth Ioo bereits von Lass[alle] empfingen, überweisen. ${ }^{2}$

Den Ladenpreis kann ich bei dem Risiko, das ich bei einem derartigen schwer wissenschaftl[ichen] Werke laufe, nicht unter einen Taler Preuss. stellen.

\section{MARX AN DUNCKER.}

Geehrter Herr,

London, 2. Juni 1859

Ich bedaure in der Tat, Ihnen einen verletzenden Brief geschrieben zu haben. Sie erlauben mir daher, in wenigen Worten meine Entschuldigungsgründe anzuführen. Einmal bin ich wirklich zu lang von Deutschland entfernt und hatte mich zu sehr an Londoner Verhältnisse gewöhnt, um den Gang des deutschen Geschäfts richtig zu würdigen. Dann aber, wie Lassalle von mir schon vor ungefähr 8 Wochen erfuhr, stehe ich in Unterhandlung mit einem Londoner Buchhändler wegen einer englischen Bearbeitung des ersten Hefts. ${ }^{3}$ Die beständig falsche

1 Von Engels bzw. Lassalle.

${ }^{2}$ Marx hatte diesen Betrag mit Schreiben Lassalles vom 8. April als Darlehn erhalten.

${ }^{3}$ Marx teilte Lassalle am 28. März mit, er "stehe in Verhandlung mit einem Engländer wegen einer englischen Bearbeitung dieser ersten Hefte." Darüber ist nichts weiter be- 
Auskunft, die ich, selbst beständig unter falschen Voraussetzungen, diesem Mann über das Erscheinen der Schrift geben musste, reichten vollständig hin, um mich in den Augen dieses John Bull als einen regular humbug blosszustellen. Die fortwährenden ungeduldigen Anfragen meiner Freunde und endlich das von einer biesigen Berliner Clique, ich weiss nicht aus welchen Motiven, sorgfältig verbreitete Gerücht, die Sache werde gar nicht erscheinen, gaben meiner Geduld den letzten Rest.

Schliesslich hoffe ich, dass Sie, in Erwägung dieser Gründe, in meinem Brief nur den hastigen Ausdruck einer durch allerlei Umstände verursachten Gereiztheit sehen und mich von aller Absicht, Sie irgendwie zu verletzen, freisprechen werden.

Ihr g[an]z ergebener

Dr. K. Marx.

8. MARX AN DUNCKER.

[Von Dunckers Hand:] beantwortet laut Cop. Buch fol. 758.

Manchester, 22. Juni I 859.

Sehr werter Herr,

Ich ersuche Sie, das restierende Honorar für meine Schrift unmittelbar meiner Frau nach London zu schicken.

In Ihrem Brief Ende Mai hiess es, dass „,nächste Woche” das Buch erscheinen und das Honorar gezahlt werden würde. Es ist weder das eine, noch das andre geschehen, bis heute, Juni 22. ${ }^{1}$ Es scheint Ihr Prinzip, dass ein „streng wissenschaftliches Werk” nicht spät genug erscheinen kann und namentlich die Epoche abgewartet werden muss, wo die Verallgemeinerung des Kriegs das "streng wissenschaftliche" Interesse belebt.

Dieselben Umstände, die Sie vermocht haben zu dem neuen Aufschub, können vielleicht einen Aufschub ins Jahr I 860 berechtigen, namentlich, da Sie mir schreiben, dass es Mode der deutschen Buchhändler ist, bei wissenschaftlichen Schriften, bei denen kein Kontrakt über die Zeit der Veröffentlichung bestebt, Embargo auf ihre Ausgabe zu legen, so oft es bequem scheint wegen des Erscheinens von Tagesschriften.

kannt. Marx hatte sich an Dana, Redakteur der New York Tribune, wegen eines Verlegers für eine englische Übersetzung gewandt. Dieser antwortete am 15. März, in Amerika sei für derartige Bücher kein Verleger zu finden; sogar Carey müsse seine Werke auf eigene Kosten drucken lassen.

${ }^{1}$ Das Werk war, was Marx nicht wissen konnte, am i r. Juni erschienen. S. den folgenden Brief. 
Da es mir unmöglich ist, den Anfragen an mich - betreffs dieser Verzögerung - privatim zu antworten, werde ich, nachdem ich noch einige Tage gewartet, eine öffentliche Erklärung erlassen.

\section{Ihr ganz ergebener}

Dr. K. Marx.

9. DUNCKER AN MARX.

Geehrter Herr!

Berlin, den 25. Juni 1859 .

Antwortlich Ihres Geehrten vom 22. c., welches mir soeben zugeht, bemerke ich einfach, dass mich von der Flut Ihrer Vorwürfe und Unterstellungen wieder nur der eine trifft, dass ich Ihnen den Rest Ihres Honorars noch nicht übersandt habe. Denn bereits am II. c. habe ich zum Zeichen, dass Ihr Buch fertig, drei Exemplare davon für Sie bestimmt den Herrn Asher \& Co. hier übergeben, welche regelmässige Sendungen an ihr dortiges Haus D. Nutt, 270 Strand machen. Sollten Ihnen diese Exemplare noch nicht zugegangen sein, so wollen Sie die Güte haben, deshalb bei N[utt] anzufragen. Ausserdem stelle ich Ihnen noch neun Freiexemplare zur Disposition, welche ich Ihnen noch nicht sende, weil Sie über einzelne derselben vielleicht hier in Deutschland $\mathrm{zu}$ verfügen haben. Die anderweite Versendung des Buches hat in der vorigen Woche begonnen und ist in dieser vollendet worden, wonach Sie also die betr. Anfragen privatim beantworten wollen, dass jetzt jede gute deutsche Buchhandlung imstande ist, das Buch zu liefern. Für Amerika haben Westermann \& Co. und Schmidt in New York Exemplare erhalten. Inliegend empfangen Sie 90 Taler in Preuss[ischen] Kassenanweisungen als Rest Ihres Honorars, dessen Empfang Sie mir bestätigen wollen. Das Heft hat nämlich elf Bogen gegeben. Sie haben daher im ganzen zu empfangen 33 Friedrichsd'or $=187$ Taler; davon empfingen Sie durch Lassalle bereits 97 Taler Is Silbergroschen und anbei 90 Taler $=187$ Taler is Silbergroschen.

Schliesslich kann ich nicht umhin zu bemerken, dass die Art Ihrer Auffassungen und der Ton Ihrer Briefe mich unmöglich auf eine Fortsetzung unserer Verbindung sehr begierig machen kann, ja es mich fast bedauern lässt, dass Lassalle überhaupt eine solche zwischen uns angeknüpft hat.

Hochachtungsvoll und ergebenst

Franz Duncker. 
IO. JENNY MARX AN DUNCKER.

[Von Dunckers Hand:] 2/7 erledigt.

London, 28. Juni 1859 .

Euer Wohlgeboren

zeige ich hiermit den Empfang des Honorars für das erste Heft der Pol[itischen] Ökonomie von 33 Friedrichsd'or an. Es ist mir nämlich unmöglich, Ihren Brief in diesem Augenblick meinem Mann zukommen zu lassen, der sich auf einer Reise durch England und Schottland befindet, Manchester aber wieder verlassen hat. Vor seiner Abreise trug er mir auf, ein Exemplar an den Oberlehrer Friedrich Köppen $z^{u}$ Berlin (Verfasser der Werke über Buddhaismus und Lamaismus) ${ }^{1}$ zu schicken, dem Sie daher dasselbe gefälligst zukommen lassen wollen. Die übrigen 8 Exemplare bitte ich hierhin zu adressieren, da diese Anzahl für die hiesigen Revuen erheischt ist.

Mit Hochachtung

Jenny Marx.

II. DUNCKER AN ENGELS.

Geehrter Herr!

Berlin, 3. August I 859 .

In ergebener Beantwortung Ihrer Zeilen vom 25. v. M. zeige ich Ihnen an, dass allerdings infolge Lassalles Aufforderung, dass Sie noch einige Exemplare wünschten, schon unterm I8. Juni für Sie an Williams \& Norgate sechs Exemplare gesandt; aber entweder hat Lassalle es vergessen Ihnen zu schreiben, oder ich, es ihm zu sagen, dass Sie dieselben dort in Empfang nehmen möchten. Ich habe solche damals noch nicht unter Ihrer Adresse abgeschickt, weil es damals noch wichtig schien, Thre Anonymität streng zu wahren, und ich daher auch mein eigenes Personal nicht auf die Fährte des Autors leiten wollte. Gegen inliegenden Schein ${ }^{2}$ werden Ihnen Williams \& Norgate die Exemplare ausliefern. Jetzt werde ich Ihnen, da ich jetzt schon wieder Exemplare zurückerhalte, die abzusetzen ich keine Aussicht mehr habe, auch noch sechs Exemplare durch Thimm ${ }^{3}$ senden.

Was den Absatz betrifft, so kann ich darüber noch wenig sagen.

${ }^{1}$ Karl Friedrich Koeppen, Marx' Studienfreund während der Berliner Zeit, veröffentlichte 1857 und 1859 ein zweibändiges Werk „Die Religion des Buddha."

${ }^{2}$ Den Schein schickte Engels am io. August an Marx mit der Bitte, sich die sechs Exemplare geben zuh lassen.

${ }^{3}$ Engels' Buchhändler in Manchester. 
Die ganze Auflage ( und viel von dem Schriftlein gesprochen worden; allein wieviel davon sich bis zur nächsten Ostermesse wieder einfinden werden, vermag ich schwer zu sagen. Es ist eben eine entsetzlich langweilige Geschichte mit dem deutschen Buchhandel. Dass die Leute (Nutt) in London keine Exemplare hatten, ist ihre eigene Schuld. Die Herren in London wollen der Fracht wegen keine unverlangten Zusendungen haben, sondern alles selbst bestellen. Nun habe ich das Schriftchen zu wiederholten Malen in unsern Buchhändlerblättern mit der Aufforderung zu bestellen angezeigt; wenn es also der eine oder der andere unterlässt, so ist das sein Versehen.

Marx' Buch werden Sie binnen kurzem nicht nur in der Augsburger [Allgemeinen Zeitung] und der Kölnischen [Zeitung], sondern in den meisten deutschen Zeitungen angekündigt finden. Bei Büchern, welche nicht sofort veralten, wirkt, aus Erfahrung weiss ich das, eine Anzeige weit mehr, wenn sie dem Erscheinen des Buches erst um einige Wochen folgt. Weil für den ersten Augenblick die Sortimenter durch AnsichtVersenden für die Bekanntmachung sorgen und sich von selbst dafür interessieren. Folgt dann nach ein paar Wochen die Ankündigung, so werden sie aufs neue an Verwendung für das Buch erinnert ${ }^{1}$.

Von der Broschüre „Po und Rhein” sind mir allerdings verschiedene, im ganzen sehr günstige Rezensionen zugekommen, doch bedaure ich, Ihnen dieselben im Augenblick wenigstens nicht senden zu können, da ich sowohl wie mein Personal gerade allzusehr beschäftigt sind, dieselben herauszusuchen. Ich werde jedoch nicht verfehlen, Ihnen dieselben gelegentlich zu übermachen, bitte dann aber nach gemachtem Gebrauch um Rücksendung, da ich die eingegangenen Rezensionen bei den Verlagsakten meiner Artikel aufzubewahren pflege.

[Die Unterschrift fehlt.]

I2. DUNCKER AN ENGELS.

Geehrter Herr!

Berlin, I 3. Februar 1860.

Ihr gef [älliges] Schreiben vom 6. (?) c. war darin nicht ganz klar, ob Sie von mir eine Antwort noch vor dem Eintreffen Ihres Manuskriptes hier erwarten oder nicht. Ich nahm zuerst das letztere an und habe Ihnen daher bis jetzt nicht geschrieben. Da mir aber eben einfällt, Sie könnten am Ende eine Antwort von mir vor Absendung des

${ }^{1}$ Engels an Marx ro. August: „Duncker will mich redlich betrügen. Lassalle schricb von 2000 Exemplaren, jetzt will er bloss roo0 gedruckt haben... Was sagst Du zu der schönen Entschuldigung der Nichtanzeige Deines Buches?” 
Manuskriptes erwarten, so beeile ich mich Ihnen, da ich gerade noch eine Viertelstunde vor Postschluss habe, zu melden, dass ich bereit bin, den Verlag der von Ihnen verfassten neuen Broschüre ${ }^{1}$ zu übernehmen unter den von Ihnen aufgestellten Bedingungen, vorausgesetzt dass ihr Umfang keinenfalls drei Bogen (Format wie „Po und Rhein”) übersteigt und dass mir der Inhalt derselben, was ich freilich nicht voraussetze, zu keinen prinzipiellen Bedenken Veranlassung gibt.

Ich sehe demnach der Einsendung des Manuskriptes entgegen. Dass Ihnen die Exemplare von „Po und Rhein" durch Thimm nicht zugegangen sind, bedaure ich recht sehr, - haben Sie denn etwa einmal bei jenem Herrn nachgefragt? - Abgesandt sind dieselben seiner Zeit von hier.

\section{Achtungsvoll und ergebenst Franz Duncker.}

\section{DUNCKER AN ENGELS.}

\section{Geehrter Herr!}

Berlin, 27. Februar 1860. Am vorigen Freitag empfing ich Ihr wertes Schreiben vom 20. c. nebst Manuskript; am Sonnabend bereits habe ich letzteres Ihrem Wunsche gemäss dem Bildhauer Herrn Afinger eingehändigt, da mich nun in der Tat prinzipielle Bedenken von der Übernahme des Verlages abhalten. Ich bin zwar keineswegs gesonnen, die Verantwortlichkeit für alle Artikel meines Verlages zu übernehmen, darin haben Sie schon recht; aber bei einer anonymen Schrift fällt allerdings in den Augen des Publikums ein gut Teil dieser Verantwortlichkeit auf den Verleger. Thre Einleitung ${ }^{2}$ greift nun gerade die Anschauung der Dinge, welche von einer Intervention zugunsten Österreichs in den italienischen Wirren von Anfang an abgeraten hat, auf das entschiedenste an. Diese Anschauung ist nun aber von der Volkszeitung sowie von mir persönlich stets vertreten worden, und es würde komisch aussehen, wenn ich mir selbst jetzt dafür gewissermassen Ohrfeigen erteilen wollte und ausserdem gegen meine Überzeugung dazu beitragen, die preuss[ische] Regierung zu einer Politik zu drängen, in

' „Savoyen, Nizza und der Rhein."

"In der Einleitung, S. 5, griff Engels das ,gothaisch-liberale Philisterium" scharf an: „Ihm war der Krieg willkommen, weil er Österreich schwächen und dadurch die endliche Eröffnung des kleindeutschen oder grosspreussischen Kaisertums möglich machen konnte. Mit ihm verband sich die Masse der norddeutschen Vulgärdemokratie, die darauf spekulierte, Louis Napoleon werde Osterreich zertrümmern und ihr dann erlauben, ganz Deutschland unter preussischer Herrschaft einig zu machen... Der stärkste der Alliierten aber war - sagen wir es offen - die Feigheit des deutschen Spiessbürgertums... Mit dieser Feigheit ging Hand in Hand die bekannte Superklugheit,... die nie ihr Jena herankommen sieht, und deren Zentralsitz Berlin ist." 
welche dieselbe hineinzureissen mir sehr entgegenstehende Richtungen nur allzusehr beschäftigt sind. Mit den Gründen für meine politischen Anschauungen will ich Sie nicht behelligen; Sie würden darin eben Berliner Kannegiessereien erblicken. Eins nur will ich noch bemerken, dass Sie die Stellung der Parteien in der auswärtigen Politik hier bei uns nicht richtig schildern; denn die Gothaer, ${ }^{1}$ soweit sie wirklich Anteil an der Regierung bei uns haben, und auch die ausserpreussischen (ich erinnere an Gagerns Brief) ${ }^{2}$ steuern weit mehr auf die österreichische Allianz hin, während Sie dieselben für die preussische Neutralitätspolitik verantwortlich machen. Zu der Partei der Nichteinmischung gehört bei uns vielmehr der grösste Teil der demokratischen, die entschiedeneren Elemente aus dem goth[aischen] Lager und ein gut Teil der sogenannten altpreussischen Partei, in welchem das Gefühl des Gegensatzes gegen Österreich noch sehr lebendig ist.

Hätten Sie nicht jede Änderung in Ihrer Broschüre auf das entschiedenste von vornherein verbeten, so würden wir übrigens trotz des Gegensatzes unserer Anschauung wohl zu einer Einigung in betreff des Verlages gekommen sein, da sich meine Ausstellungen fast nur auf Ihre Einleitung beziehen, die mit dem Kern Ihrer Schrift nicht viel zu tun hat, und ich mir dagegen Ihre Schlussbetrachtungen, ${ }^{3}$ mit denen ich zwar auch nicht übereinstimme, wohl hätte gefallen lassen können.

\author{
Achtungsvoll und ergebenst \\ Franz Duncker.
}

\begin{abstract}
${ }^{1}$ Der Nationalverein, Nachfolger der sogen. Professorenpartei des "Casino" von I848; aus ihm gingen die meisten Führer der nationalliberalen Partei hervor.

${ }^{2}$ Heinrich von Gagern vertrat als Präsident der Nationalversammlung als Ziel den deutschen Bundesstaat unter preussischer Führung, aber in Union mit dem abgetrennten Österreich. Seitdem hatte er seine Ansicht geändert. In einem Brief an Max Duncker äusserte er sich sehr heftig über die Haltung der preussischen Regierung. Er war auf den Ton gestimmt: ,...Wie Preussen mit solchem Hass gegen Österreich im Herzen...in Deutsehland moralische Eroberungen zu machen gedenken mag - das grenzt an Blödsinn." M. Duncker, Politischer Briefwechsel aus seinem Nachlass, Stuttgart u. Berlin 1923, S. I04.

${ }^{3}$ Den Nachdruck legte Engels in den politischen Betrachtungen seiner Broschüre auf die Rolle det zaristischen Politik. Er zählte, S. 45 ff., alle aggressiven, feindseligen Handlungen Russlands gegenüber Preussen und Österreich vom Jahre $x 807$ an auf, die Deutschland niemals vergessen dürfe. ,Mit dem Russland, das von Peter dem Grossen bis Nikolaus bestand, fällt auch die auswärtige Politik dieses Russland. Wie es den Anschein hat, ist es Deutschland vorbehalten, diese Tatsache den Russen nicht nur mit der Feder, sondern auch mit dem Schwert klarzumachen. Kommt es dabin, so ist das eine Rehabilitation Deutschlands, die Jahrhunderte politischer Schmach aufwiegt."
\end{abstract}

